\title{
Method comparison of three serum free light chain assays on the Roche Cobas 6000 c501 chemistry analyzer
}

https://doi.org/10.1515/cclm-2021-1029

Received September 21, 2021; accepted December 13, 2021; published online December 30, 2021

\section{Abstract}

Objectives: Free light chains (FLC) are important in the diagnosis, prognosis and monitoring of therapy response of patients with monoclonal gammopathies. In this study, we performed a method comparison of three FLC assays on the Cobas 6000 c501 chemistry analyzer of Roche Diagnostics.

Methods: Samples of 119 patients with various monoclonal gammopathies and 26 control patients were measured with the Freelite (The Binding Site), Diazyme (Diazyme Laboratories) and KLoneus (Trimero Diagnostics) FLC assays. A method comparison was performed and reference intervals of the three assays were validated.

Results: The analysis of the Bland-Altman agreement showed bias between the three FLC assays, ranging from -62.7 to $5.1 \%$ for $\kappa$ FLC and between -29.2 to $80.5 \%$ for $\lambda F L C$. The Freelite and Diazyme assays have the highest agreement. The concordance of the FLC-ratio ranges from 41 to $75 \%$, with the highest concordance between the Freelite and KLoneus assays. The FLC-ratio in 25 sera from healthy controls were within the reference ranges of the Freelite and KLoneus assays. The FLC-ratio was elevated in all 25 samples tested with the Diazyme assay.

Conclusions: The agreement for the free light chains is highest between the Freelite and the Diazyme assay and fair for the KLoneus assay. However, concordance of the FLC-ratio is highest when the Freelite and KLoneus assays were compared. Our data suggest that concordance for the

\footnotetext{
*Corresponding author: Dr. Henk Russcher, Department of Clinical Chemistry, Erasmus MC, University Medical Center Rotterdam, Dr. Molewaterplein 40, Room Na407, 3015 GD Rotterdam, The Netherlands, Phone: +31 (0)10 7033581,

E-mail: h.russcher@erasmusmc.nl

Dieuwertje Augustijn, Department of Clinical Chemistry, Erasmus MC, University Medical Center Rotterdam, Rotterdam, The Netherlands. https://orcid.org/0000-0002-7990-3661

Joannes F.M. Jacobs, Department of Laboratory Medicine, Radboud University Medical Center, Nijmegen, The Netherlands
}

Diazyme assay could be improved by recalibration. Because of absolute differences between the three methods in individual patients, none of the three FLC assays can be used interchangeably.

Keywords: free light chains; M-protein; monoclonal gammopathy; multiple myeloma.

\section{Introduction}

Monoclonal gammopathies are characterized by clonal proliferation of plasma cells leading to the production of monoclonal immunoglobulins, the so-called M-proteins. Plasma cells also secrete free light kappa $(\kappa)$ or lambda $(\lambda)$ which circulate unbound to the immunoglobulin heavy chains. In plasma cell proliferative disorders, there is often excess production of one of the light chains, leading to an abnormal free light chain (FLC) ratio $(\kappa / \lambda)$. FLC are important in the diagnosis, prognosis and monitoring of therapy response $[1,2]$. About $90 \%$ of the newly diagnosed multiple myeloma (MM) patients have an abnormal FLC-ratio and $70 \%$ of patients with smoldering multiple myeloma [1]. Because it was shown that $80 \%$ of patients with an involved/uninvolved FLC-ratio $>100$ progresses to MM within 2 years, the International Myeloma Working Group defined that FLC-ratio as a myeloma defining event [1].

Since the introduction of serum free light chain in the early 2000s, several immunoassays have been introduced that make use of nephelometry, turbidimetry, ELISA or lateral-flow methodology [3-6]. It is known that these different FLC assays can lead to different outcomes, especially in terms of absolute FLC quantification. Harmonization of FLC-quantification over different assays has proven to be challenging because of the unique characteristics of the analyte and because of the lack of a reference method or reference material [7-10].

On the Cobas analyzer series of Roche Diagnostics, there are three different immunoassays available using turbidimetry, namely the Freelite assay of the Binding Site, the Diazyme assay of Diazyme Laboratories and the KLoneus assay of Trimero Diagnostics. The three assays are available on the c501 module of the Cobas 6000 and for the 
c502 module of the Cobas 8000 . The advantage of these immunoassays on the Cobas analyzer series is that it allows automated continuous analysis which results in higher reproducibility and short turnaround times.

All three immunoassays make use of polyclonal antibodies, but there is a difference in assay principle. Both Freelite and Diazyme assays are latex-enhanced turbidimetric immunoassays. In these assays, latex particles are coated with antibodies against either free light chain kappa or lambda. The KLoneus assay is using polystyrene particles coated with FLC-specific antibodies. The reaction between the antibodies bound to particles from the assay reagents and the FLCs in the patient samples forms insoluble particles that change the intensity of the transmitted light due to the scattering effect of the particles. Changes in the intensity of the transmitted light are measured using turbidimetry.

The Binding Site has sent out a notification that the supply of Freelite reagents for the Roche Cobas analyzers will not be continued in the near future. If users would like to continue using the Roche Cobas analyzer series, they could switch to the Diazyme or the KLoneus assay. The purpose of this study is to compare the three currently available FLC assays on the Roche Cobas c501 chemistry analyzer.

\section{Materials and methods}

\section{Free light chain assays}

Serum free light chain measurements were performed on the Cobas 6000 c501 using Freelite (reagent lot number 712007 for FLC kappa and 711571 for FLC lambda, The Binding Site, Birmingham, UK), Diazyme (reagent lot number 491684 for FLC kappa and 479603 for FLC lambda, Diazyme Laboratories, Poway, USA) and the KLoneus assay (reagent lot number 511SK-54 for FLC kappa and 511SL-54 for FLC lambda, Trimero Diagnostics, Barcelona, Spain) according to the manufacturer's guidelines. Characteristics of the different sFLC assays are presented in Table 1.

\section{Samples}

In this study, 145 patients are evaluated, which were used in a previous method comparison of different free light chain assays [10]. This includes 119 patients with monoclonal gammopathies, including multiple myeloma (MM, $\mathrm{n}=62$ of which five newly diagnosed patients), kappa light chain multiple myeloma (LCMM, $n=19$ ), lambda LCMM $(\mathrm{n}=8)$, smoldering multiple myeloma ( $\mathrm{sMM}, \mathrm{n}=7)$, AL amyloidosis ( $n=13$ of which two newly diagnosed patients), Waldenström macroglobulinemia $(n=5)$ and MGUS $(n=5)$. The diagnosis was performed according to the criteria of the International Myeloma Working Group [1], including performing immunofixation of serum and/or urine. The patients were not treated with monoclonal antibodies during collection of the samples. In total, 26 control patients are included from patients without M-protein ( $\mathrm{n}=11)$ and patients with chronic kidney

Table 1: Characteristics of the FLC assays on the Cobas 6000 c501.

\begin{tabular}{|c|c|c|c|}
\hline & Freelite & Diazyme assays & KLoneus assay \\
\hline Assay principle & $\begin{array}{l}\text { Latex-enhanced turbidimetric } \\
\text { immunoassay }\end{array}$ & $\begin{array}{l}\text { Latex-enhanced turbidimetric } \\
\text { immunoassay }\end{array}$ & $\begin{array}{l}\text { Particle-enhanced turbidimetric } \\
\text { immunoassay }\end{array}$ \\
\hline Antibodies & Polyclonal & Polyclonal & Polyclonal \\
\hline Calibrator & Polyclonal FLC & Polyclonal FLC & Polyclonal FLC \\
\hline Sample volume & $\begin{array}{l}\kappa \mathrm{FLC}: 20 \mu \mathrm{L} \\
\lambda \mathrm{FLC}: 20 \mu \mathrm{L}\end{array}$ & $\begin{array}{l}\kappa \mathrm{FLC}: 6.5 \mu \mathrm{L} \\
\lambda \mathrm{FLC}: 13 \mu \mathrm{L}\end{array}$ & $\begin{array}{l}\kappa \mathrm{FLC}: 3 \mu \mathrm{L} \\
\lambda \mathrm{FLC}: 5 \mu \mathrm{L}\end{array}$ \\
\hline $\begin{array}{l}\text { Intra-assay VC } \\
\text { (within-run precision) }\end{array}$ & $1.4-5.5 \%$ & $1.1-6.5 \%$ & $3.5-7.6 \%$ \\
\hline Interassay VC & $2.3-10.1 \%$ & $1.1-114.4 \%$ & $1.2-4.3 \%$ \\
\hline Reference values $k$ & $3.3-19.4 \mathrm{mg} / \mathrm{L}$ & $2.37-20.73 \mathrm{mg} / \mathrm{L}$ & $3.3-19.4 \mathrm{mg} / \mathrm{L}$ \\
\hline Reference values $\lambda$ & $5.7-26.3 \mathrm{mg} / \mathrm{L}$ & $4.23-27.69 \mathrm{mg} / \mathrm{L}$ & $5.7-26.3 \mathrm{mg} / \mathrm{L}$ \\
\hline Reference values $\kappa / \lambda$ & $0.26-1.65$ & $0.22-1.74$ & $0.26-1.65$ \\
\hline Adj. FLC-ratio & Yes, $0.37-3.1^{\mathrm{a}}$ & No & No \\
\hline Interference ${ }^{b}$ & $\begin{array}{l}\text { Bilirubin: } 150 \mathrm{mg} / \mathrm{L} \text { for kappa } \\
\text { and } 200 \mathrm{mg} / \mathrm{L} \text { for lambda } \\
\text { Hemoglobin: } 4.8 \mathrm{~g} / \mathrm{L}\end{array}$ & $\begin{array}{l}\text { Triglycerides: } 10.000 \mathrm{mg} / \mathrm{L} \\
\text { Bilirubin: } 400 \mathrm{mg} / \mathrm{L} \\
\text { Hemoglobin: } 10 \mathrm{~g} / \mathrm{L} \\
\text { Rheumatoid factor: } \\
100 \mathrm{IU} / \mathrm{mL} \\
\text { Ascorbic acid: } 10 \mathrm{mmol} / \mathrm{L}\end{array}$ & $\begin{array}{l}\text { Triglycerides: } 5,000 \mathrm{mg} / \mathrm{L} \text { for kappa } \\
\text { and } 10.000 \mathrm{mg} / \mathrm{L} \text { for lambda } \\
\text { Bilirubin: } 200 \mathrm{mg} / \mathrm{L} \\
\text { Hemoglobin: } 5 \mathrm{~g} / \mathrm{L}\end{array}$ \\
\hline Company & The Binding Site & Diazyme Laboratories & Trimero Diagnostics \\
\hline
\end{tabular}

${ }^{a}$ Adjusted $\kappa / \lambda$ FLC-ratio reference values for patients with impaired renal function, not included in the package insert but obtained from communication. ${ }^{b}$ Substances normally present in serum which produced less than $10 \%$ deviation when tested at levels equal to the concentrations listed. FLC, free light chain; VC, variation coefficient. 
disease without M-protein ( $\mathrm{n}=15)$. The samples were collected in $8.5 \mathrm{~mL}$ SST vacutainers (Becton Dickinson, UK) and separated within $1 \mathrm{~h}$ upon arrival at the laboratory. Samples were stored at $-20{ }^{\circ} \mathrm{C}$ until analysis and thawed prior to analysis. An additional 25 reference sera were derived from healthy controls for the validation of the reference intervals of the three assays. The healthy adult controls were volunteers from our laboratory, who had no active disease and were not on medication.

The study was performed in according to the Helsinki guidelines and was approved by the institutional Medical Ethics Review Board (Erasmus MC 2017-415).

\section{sFLC method comparison}

Method comparison between the three different FLC assays on the Cobas 6000 c501 were performed according to the CLSI EP9 guidelines. The entire dynamic range of FLC concentrations was covered by the 145 selected serum samples. The reference ranges from the package inserts were used (see Table 1). The adjusted FLC-ratio was used in the case of patients with renal impairment defined as eGFR $<60 \mathrm{~mL} / \mathrm{min} / 1.73 \mathrm{~m}^{2}$ using creatinine concentration. The reference interval for creatinine is $55-90 \mu \mathrm{mol} / \mathrm{L}$ for females and $65-115 \mu \mathrm{mol} / \mathrm{L}$ for males (Roche Diagnostics, Almere, The Netherlands). The FLC-ratio $(\kappa / \lambda)$ is calculated and divided into three groups: below (low), above (high) or within the reference range (normal). In patients with renal impairment, adjusted reference ranges (if applicable) were applied to categorize the FLC-ratio.

\section{Validation of reference intervals}

Validation of the reference intervals mentioned in the kit-inserts of all three methods was performed based on CLSI C28-A3 guidelines [11]. Reference samples from 25 healthy volunteers were measured with the three methods. The percentage of results that fell within the reference limit of each test was determined. When $\geq 90 \%$ of these samples fell within the reference interval the reference intervals were considered as verified.

\section{Statistics}

Bland-Altman evaluation, Passing-Bablok regression and Kendall's Tau correlation coefficient analysis were performed with the results for $\kappa$ FLC and $\lambda$ FLC obtained in three FLC assays. Nonparametric methods were used since the results are not normally distributed. The results of the categorized FLC-ratio $(\kappa / \lambda)$ were used to perform concordance analysis. The Cohen kappa coefficient $(\kappa)$ is used to interpret the concordance analysis. Values between 0.81 and 1.00 are defined as almost perfect agreement, between 0.61 and 0.80 as high agreement, between 0.41 and 0.60 as moderate agreement, between 0.21 and 0.40 as fair agreement [12]. A $\kappa$-value less than 0.20 is defined as none to slight agreement. Statistical analysis was performed using Analyse-IT for Microsoft Excel (v5.40, Analyse-IT Software Ltd., Leeds, UK).

\section{Results}

\section{Method comparisons}

Three different FLC assays were used to measure 119 sera from patients with monoclonal gammopathies and 26 sera from control patients without M-protein. In Table 2, the agreement of $\kappa$ FLC, $\lambda$ FLC and FLC-ratio are presented by Bland-Altman and Passing-Bablok for all combinations. Graphical representation of the Bland-Altman and PassingBablok analysis of $\kappa$ FLC and $\lambda$ FLC are represented in Supplementary Figure 1 and Supplementary Figure 2. Results of the FLC-ratio are represented in Figure 1.

The bias between the Freelite and Diazyme assays is $5.1 \%$ and $-29.2 \%$ for kappa and lambda, respectively, according to the Bland-Altman evaluation. The agreement of these two methods is quite high, especially for kappa. The slopes of the Passing-Bablok regression for Freelite vs. Diazyme were 1.05 (Kendall's $\tau=0.888, \mathrm{p}<0.001$ ) and 0.96 (Kendall's $\tau=0.869, \mathrm{p}<0.001$ ) for kappa and lambda, respectively. The bias between the Freelite and Diazyme assays is $30.9 \%$ for the FLC-ratio. The Passing-Bablok regression slope is 1.47 (Kendall's $\tau=0.761, \mathrm{p}<0.001$ ). Figure 1A shows higher elevated FLC-ratios when using the Diazyme assay. For the Freelite vs. KLoneus assays, there is a bias of $-56.8 \%$ for kappa and $54.9 \%$ for lambda which results in less agreement between these two assays. The slope was 0.51 (Kendall's $\tau=0.847, \mathrm{p}<0.001$ ) for kappa and 1.33 (Kendall's $\tau=0.605, \mathrm{p}<0.001$ ) for lambda when comparing Freelite with the KLoneus assay. The bias for the FLC-ratio is $-99.3 \%$ for these two assays. The slope of the Passing-Bablok regression is 0.221 (Kendall's $\tau=0.703$, $\mathrm{p}<0.001)$. Comparing the Diazyme with the KLoneus assay, there is a bias of $-62.7 \%$ for kappa and $80.5 \%$ for lambda. The slope for this comparison was 0.54 (Kendall's $\tau=0.900$,

Table 2: Bland-Altman, Passing-Bablok (PB) and Kendall's Tau ( $\tau$ ) analysis for kappa and lambda free light chains.

\begin{tabular}{|c|c|c|c|c|c|c|c|c|c|}
\hline & \multicolumn{3}{|c|}{ FLC Kappa } & \multicolumn{3}{|c|}{ FLC Lambda } & \multicolumn{3}{|c|}{ FLC-ratio } \\
\hline & Bias, \% & PB slope & Kendall's $\tau$ & Bias, \% & PB slope & Kendall's $\tau$ & Bias, \% & PB slope & Kendall's $\tau$ \\
\hline Freelite vs. Diazyme & 5.1 & 1.05 & 0.888 & -29.2 & 0.96 & 0.869 & 30.9 & 1.47 & 0.761 \\
\hline Freelite vs. KLoneus & -56.8 & 0.51 & 0.847 & 54.9 & 1.33 & 0.605 & -99.3 & 0.221 & 0.703 \\
\hline Diazyme vs. KLoneus & -62.7 & 0.54 & 0.900 & 80.5 & 1.39 & 0.666 & -123.9 & 0.156 & 0.755 \\
\hline
\end{tabular}



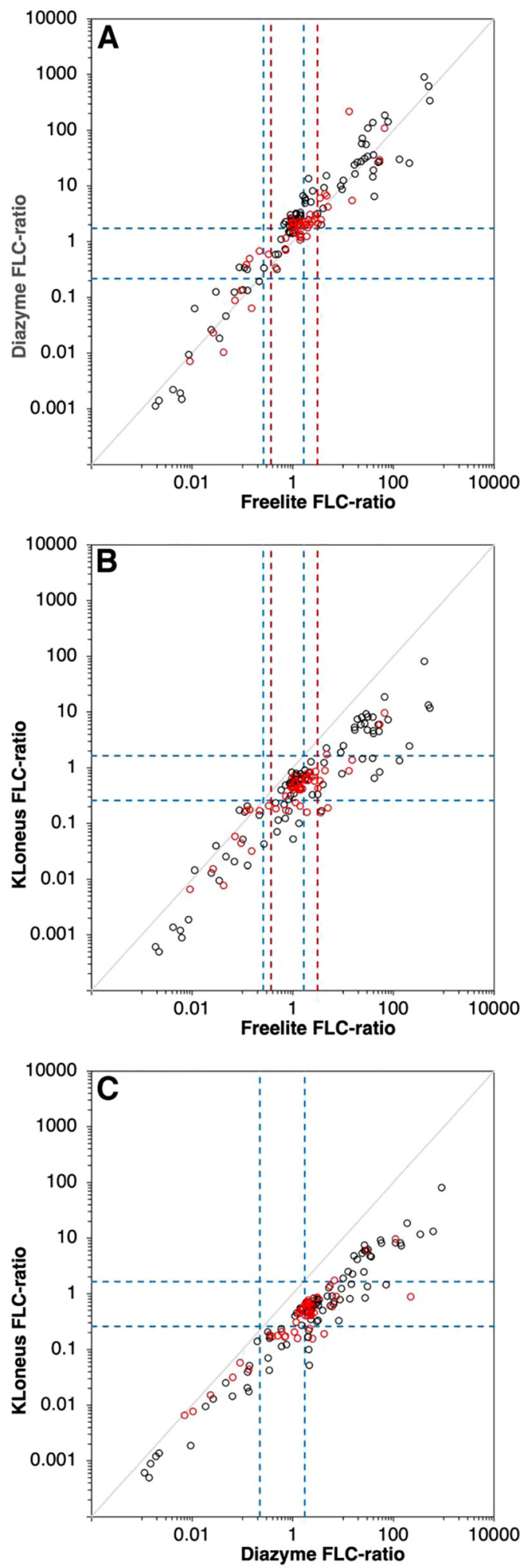

Figure 1: FLC-ratio of the 145 samples between the assays. Freelite vs. Diazyme (A), Freelite vs. KLoneus (B) and Diazyme vs. KLoneus (C). The blue dotted lines are the reference limits of the corresponding assay, the red dotted lines are the adjusted reference limit for patients with renal impairment. Values from patients with renal impairment are represented as red dots.
Table 3: FLC kappa concentration for three discrepant samples when comparing the Freelite and Diazyme assay.

\begin{tabular}{lrrr}
\hline Diagnosis & \begin{tabular}{r} 
Freelite \\
FLC kappa, \\
\cline { 3 - 4 }
\end{tabular} & \multicolumn{2}{c}{ Diazyme FLC kappa, mg/L } \\
\cline { 3 - 4 } & 625.0 & $\begin{array}{r}\text { Original } \\
\text { concentration }\end{array}$ & $\begin{array}{r}\text { Manually } \\
\text { diluted (1:50) }\end{array}$ \\
\hline MM & 83.15 & 739.5 \\
MM & 407.8 & 131.75 & 216.5 \\
MM FLC kappa & 231.4 & 67.82 & 334.5 \\
\hline
\end{tabular}

The samples are measured again after a 50-time dilution to reveal whether there is a high-dose hook effect.

$\mathrm{p}<0.001$ ) for kappa and 1.39 (Kendall's $\tau=0.666, \mathrm{p}<0.001$ ) for lambda. For the FLC-ratio, the bias is $-123.9 \%$ with a Passing-Bablok regression slope of 0.156 (Kendall's $\tau=0.755, \mathrm{p}<0.001)$. The analysis is also performed on the results of the multiple myeloma patients only, but no large differences were detected compared to the all patients with monoclonal gammopathies (Supplementary Table 1).

When inspecting the results of the Passing-Bablok analysis of the comparison between the Freelite assay and the Diazyme assay, there are some samples with discrepancy. These samples have high concentrations of FLC kappa in the Freelite assay, but low concentrations of FLC kappa in the Diazyme assay. Therefore, we diluted three samples manually and higher concentrations of FLC kappa were measured with the Diazyme assay, indicating a highdose hook effect (Table 3).

\section{Concordance analysis FLC-ratio}

For the concordance analysis, the FLC-ratios $(\kappa / \lambda)$ are classified according to the kit-insert into three groups: below (low), above (high) or within the reference range (normal) (Figure 2).

The concordances for the Freelite and Diazyme assay is $63 \%$ and a Cohen's coefficient of 0.43 , corresponding to a moderate agreement (Figure 2A). Most of the mismatches were observed in samples that were classified as normal with the Freelite assay, but high with the Diazyme assay. There was good agreement between the Freelite and the KLoneus assay (Cohen's $\kappa=0.61$ ) with a concordance of $75 \%$. Most of the discrepant samples are below the reference interval of the FLC-ratio of the KLoneus assay. When comparing the results of the FLC-ratio of the Diazyme method with the KLoneus assay, there is none to slight agreement (Cohen's $\kappa=0.20$ ) with a concordance of $41 \%$. Most of the samples are classified as normal or high FLC-ratio with the Diazyme assay, but with lower FLC-ratios with the KLoneus assay. When concordance 
A

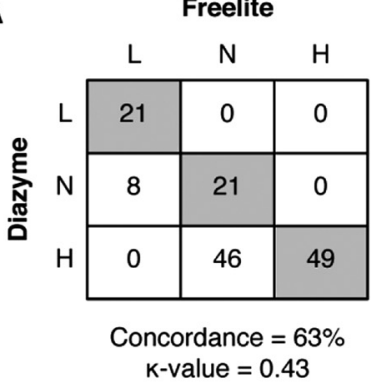

B

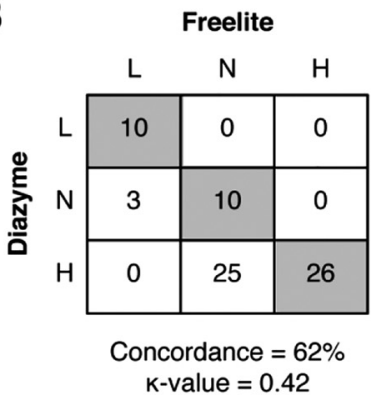

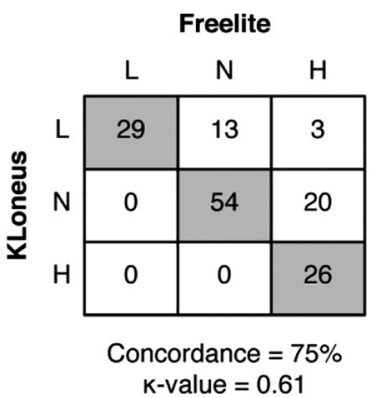

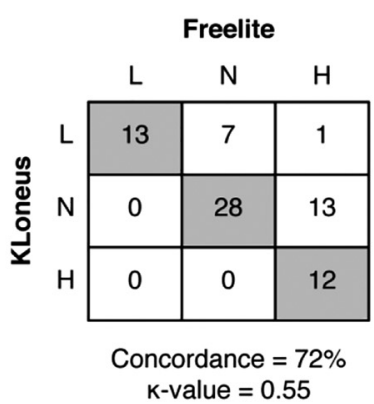

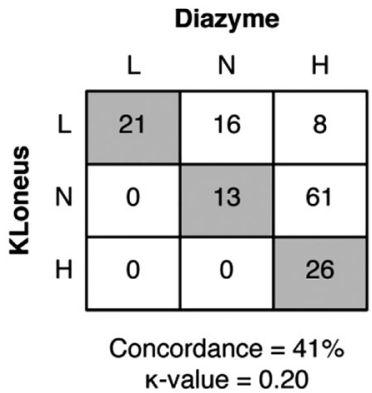

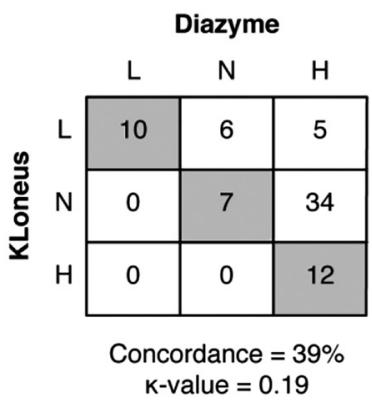

Figure 2: Concordance analysis of the FLC-ratio between the three FLC assays.

(A) For all patients $(n=145)$ and $(B)$ for patients without renal impairment $(n=74)$. Grey boxes indicate agreement between the assays. The concordance is represented in percentages, and the Cohen kappa coefficient is also displayed.

analysis is performed in patients without renal impairment (eGFR $>60 \mathrm{~mL} / \mathrm{min} / 1.73 \mathrm{~m}^{2}$ ) only, there are some minor changes in concordance and agreement between the methods (Figure 2B). The results of the FLC-ratio of the Diazyme method are still higher compared to the Freelite assay, and the results of the FLC-ratio are lower with the KLoneus assay in patients without renal impairment. The concordance analysis is also performed on multiple myeloma patients only, there are minor changes in the concordances (Supplementary Table 1).

\section{Validation of reference intervals}

Both kappa and lambda concentration were measured with the three methods in sera from 25 healthy volunteers. Table 4 shows the number and percentage of the samples which fell within the reference intervals. Validation was considered successful when $\geq 90 \%$ of the reference samples fell within the reference interval. The reference interval for both the Freelite method and the KLoneus method can be verified (Supplementary Table 2). In contrast with that, all healthy volunteers have an elevated FLC-ratio when tested with the Diazyme assay. The reason for this increased FLC-ratio is that the Diazyme assay consistently measured FLC kappa values in the upper-range of normal (and $24 \%$ of samples above). Additionally, the FLC lambda values were consistently in the lower range of normal (and 12\% of samples below).
Table 4: Validation of assay-specific reference intervals.

\begin{tabular}{lrrr}
\hline & Freelite & Diazyme & KLoneus \\
\hline FLC Kappa & $0 / 23 / 2(92 \%)$ & $0 / 19 / 6(76 \%)$ & $0 / 25 / 0(100 \%)$ \\
FLC Lambda & $0 / 25 / 0(100 \%)$ & $3 / 22 / 0(88 \%)$ & $0 / 25 / 0(100 \%)$ \\
FLC-ratio & $0 / 25 / 0(100 \%)$ & $0 / 0 / 25(0 \%)$ & $0 / 25 / 0(100 \%)$ \\
\hline
\end{tabular}

The number of samples that were below, within or above the reference limits as provided by the kit-inserts. The percentage is the number of samples which fell within the reference limit.

\section{Discussion}

The aim of this study was to perform a method comparison of three FLC assays that are currently available for routine diagnostics on the Roche Cobas automated platforms. Free light chains are important in the context of screening, prognostic stratification, and monitoring of patients with monoclonal gammopathies. The assays are all using polyclonal antibodies and turbidimetry as detection method but differ in assay principle. In this study we demonstrate that these assays are not adequately harmonized. As a consequence, the FLC-ratio test-result of individual patients may be normal or abnormal depending on the FLC assay that is used. This is also important for the involved:noninvolved FLC-ratio (iFLC/ niFLC) which is defined as $\geq 100$ by the International Myeloma Working Group (IMWG) as a criterium to start treatment for multiple myeloma patients [13]. 
In addition, the reference interval for the Diazyme assay cannot be verified. All of the 25 tested healthy control sera had an abnormal high FLC-ratio which was caused by consistently high FLC kappa results combined with consistently low FLC lambda results. From our data it seems that a simple re-calibration of both FLC kappa and FLC lambda could solve this issue. This is also evident in the high level of agreement between the Freelite and Diazyme when comparing the FLC kappa and lambda to each other. The bias for FLC kappa is only $-5.1 \%$ and for FLC lambda $-29.2 \%$. However, the concordance between the two assays for the FLC-ratio is low with only $63 \%$ due to the low reference-interval for FLC lambda. In addition, we showed for three samples that the discrepancy of the results of the FLC kappa concentrations can be explained by a high-dose hook effect.

The agreement between the Freelite and KLoneus assay was low. The concentration of kappa FLC was lower with the KLoneus assay (bias $=-56.8 \%$ ) and a higher concentration of lambda FLC (bias $=54.9 \%$ ) compared to the Freelite assay. However, the concordance of the FLC-ratio was highest with $75 \%$ agreement between the Freelite and KLoneus assay. Although calibration for both assays was performed by using the Freelite calibrator, this didn't result in a high agreement between the two assays and could be affected by different assay antibodies that recognize different variations [14]. The agreement between Diazyme and the KLoneus assay was low as well with a bias of $-62.7 \%$ for kappa FLC and $80.5 \%$ for lambda FLC. There was also none to slight agreement when comparing the FLC-ratio between the two assays. A limitation of this study is that the cohort is too small to perform reliable method comparisons stratified per individual monoclonal gammopathy.

Our data show that a large part of the differences between the three assays can be explained by a calibrationissue. Recalibration (especially of the Diazyme assay) to the Freelite assay can solve a large part of the discrepancies. For the KLoneus assays, we observe excellent concordance in healthy control sera between both assays, indicating perfect calibration to the Freelite assay in the reference range. However, when monoclonal free light chains are present, significant differences between FLC-quantification was observed exposing intrinsic differences between the Freelite and KLoneus FLC-assays [15].

Other discrepancies between the three assays were caused by FLC antigen excess that was not properly detected and possible differences of the various detection reagents to measure polymerized FLC $[16,17]$ or rare FLC-epitopes [7].

It is known that renal impairment influences renal clearance of both FLC kappa and lambda [18]. In some of the FLC-assays also an increased FLC-ratio is observed in these patients [10]. The Freelite assay has an adjusted reference interval of the FLC-ratio for patients with renal impairment. KLoneus and Diazyme have not communicated the need for adjusted reference ranges in these patients, however our data seem to indicate that the Diazyme Laboratories needs to introduce adjusted reference ranges for patients with renal impairment as well. Our cohort is too small to determine the exact ranges for these adjusted reference ranges.

In conclusion, the agreement between the kappa and lambda FLC Freelite assay of The Binding Site and the Diazyme assay of Diazyme Laboratories were good in this method comparison. There was a moderate agreement when looking at the FLC-ratio. Our data strongly indicate that the Diazyme assay needs to be recalibrated, since the FLC-ratio in all healthy controls tested in this study were slightly above the upper limit of normal. The Trimero Diagnostics KLoneus assays show poor agreement with both the Freelite assay and the Diazyme assay.

Acknowledgments: Reagents to perform this study were kindly provided by The Binding Site, Roche Diagnostics $\mathrm{GmbH}$ and Trimero Diagnostics.

Research funding: JFMJ received a research grant from the Dutch Cancer Society (\#10817).

Author contributions: All authors have accepted responsibility for the entire content of this manuscript and approved its submission.

Competing interests: Authors state no conflict of interest. Informed consent: Not applicable

Ethical approval: The study was performed in according to the Helsinki guidelines and was approved by the institutional Medical Ethics Review Board (Erasmus MC 2017-415).

\section{References}

1. Rajkumar SV, Dimopoulos MA, Palumbo A, Blade J, Merlini G, Mateos MV, et al. International Myeloma Working Group updated criteria for the diagnosis of multiple myeloma. Lancet Oncol 2014; 15:e538-48.

2. Graziani MS, Merlini G. Serum free light chain analysis in the diagnosis and management of multiple myeloma and related conditions. Expert Rev Mol Diagn 2014;14:55-66.

3. Bradwell AR, Carr-Smith HD, Mead GP, Tang LX, Showell PJ, Drayson MT, et al. Highly sensitive, automated immunoassay for immunoglobulin free light chains in serum and urine. Clin Chem 2001;47:673-80.

4. te Velthuis H, Knop I, Stam P, van den Broek M, Bos HK, Hol S, et al. N Latex FLC - new monoclonal high-performance assays for the determination of free light chain kappa and lambda. Clin Chem Lab Med 2011;49:1323-32.

5. Campbell JP, Heaney JL, Shemar M, Baldwin D, Griffin AE, Oldridge $E$, et al. Development of a rapid and quantitative lateral flow assay 
for the simultaneous measurement of serum $\kappa$ and $\lambda$ immunoglobulin free light chains (FLC): inception of a new nearpatient FLC screening tool. Clin Chem Lab Med 2017;55:424-34.

6. Jacobs JFM, de Kat Angelino CM, Brouwers H, Croockewit SA, Joosten I, van der Molen RG. Evaluation of a new free light chain ELISA assay: bringing coherence with electrophoretic methods. Clin Chem Lab Med 2018;56:312-22.

7. Graziani MS. Measurement of free light chains - pros and cons of current methods. Clin Chem Lab Med 2016;54:1015-20.

8. Jacobs JF, Tate JR, Merlini G. Is accuracy of serum free light chain measurement achievable? Clin Chem Lab Med 2016;54:1021-30.

9. Cigliana G, Gulli F, Napodano C, Pocino K, De Santis E, Colacicco L, et al. Serum free light chain quantitative assays: dilemma of a biomarker. J Clin Lab Anal 2018;32. https://doi.org/10.1002/jcla. 22243.

10. Fleming CKA, Swarttouw T, de Kat Angelino CM, Jacobs JFM, Russcher $\mathrm{H}$. Method comparison of four clinically available assays for serum free light chain analysis. Clin Chem Lab Med 2019;58:85-94.

11. CLSI I. Defining, establishing, and verifying reference Intervals in the clinical laboratory; Approved Guideline - third edition: book title CLSI document EP28-A3c. Wayne, PA: Clinical and Laboratory Standards Institute; 2008.

12. McHugh ML. Interrater reliability: the kappa statistic. Biochem Med 2012;22:276-82.
13. Curcio R, Stettler H, Suter PM, Aksözen JB, Saleh L, Spanaus K, et al. Reference intervals for 24 laboratory parameters determined in 24-hour urine collections. Clin Chem Lab Med 2016;54:105-16.

14. Tate J, Bazeley S, Sykes S, Mollee P. Quantitative serum free light chain assay-analytical issues. Clin Biochem Rev 2009;30:131-40.

15. Carr-Smith HD, Jenner EL, Evans JA, Harding SJ. Analytical issues of serum free light chain assays and the relative performance of polyclonal and monoclonal based reagents. Clin Chem Lab Med 2016;54:997-1003.

16. Caponi L, Koni E, Romiti N, Paolicchi A, Franzini M. Free light chain UV quantification compared with immunochemical measurement: how dimers and monomers may influence the results. Clin Chim Acta 2020;510:278-84.

17. Kaplan B, Jacobs JFM. FLC polymerization: another hurdle towards standardization of FLC measurements. Clin Chim Acta 2021;515: 42-3.

18. Molina-Andújar A, Robles P, Cibeira MT, Montagud-Marrahi E, Guillen $E$, Xipell $M$, et al. The renal range of the $\kappa / \lambda$ sFLC ratio: best strategy to evaluate multiple myeloma in patients with chronic kidney disease. BMC Nephrol 2020;21:111.

Supplementary Material: The online version of this article offers supplementary material (https://doi.org/10.1515/cclm-2021-1029). 